\title{
Variación en pequeña escala de la estructura horizontal y vertical de comunidades macrobentónicas en una laguna costera del Atlántico Sudoccidental
}

\author{
Small-scale variation of the horizontal and vertical \\ structure of macrobenthic communities in a coastal \\ lagoon of the southwestern Atlantic
}

RECIBIDO: 20/2/2019 $\rightarrow$ APROBADO: 20/5/2019 ワjeny2517@gmail.com

\begin{abstract}
i̊ pereira, Jennifer (1); Rodríguez, matilde (1); SAntos, sofía (1); Kandratavicius, Noelia (1); BUeno, CARolina (1); Rodríguez, MarCel (1); PÉREZ, LAURA (2); TUdURí, AdRiana (1); VENTURINi, NATAlia (1); BRUGNOLI, ERNESTO (1); MUNIZ, PABLO (1).
\end{abstract}

(1) oceanografía y eCología marina, Instituto de ECología y Ciencias Ambientales (IECA), FACUltad DE CIENCIAS, UNiversidad DE LA REPÚBlicA, MONTEVIDEO, URUGUAY.

(2) Centro universitario regional este (Cure), universidad de la república, ROCHA, URUGUAY.

\section{RESUMEN}

La Laguna de Rocha forma parte de la cadena de lagunas costeras que se extienden por la costa atlántica uruguaya y se continúa hacia el sur de Brasil. Este sistema presenta una dinámica ecológica compleja debido al gradiente marino-salobre. La macrofauna bentónica, componente importante de estos ambientes estuariales, se ve afectada por la compleja dinámica del sistema. Se analizó cómo afectan las lenguas de arena (acumulaciones de arena perpendiculares a la barra) a la fauna macrobentónica. Se buscó establecer la relación del macrobentos con variables ambientales a una escala vertical (estratos dentro del sedimento) y horizontal (a un lado y al otro de las lenguas), y evaluar la influencia de la distancia a la intrusión marina. En todos los puntos de muestreo los parámetros ambientales (materia orgánica, fósforo total, oxígeno disuelto, clorofila- $a$ y feopigmentos) presentaron una tendencia vertical (disminución hacia el fondo) y el macrobentos se relacionó con estos cambios. Se observó la dominancia de Heleobia australis, Nephtys fluviatilis y Heteromastus similis en diferentes estaciones y estratos. Existe una partición 
espacial de los recursos reflejada por estos cambios de dominancia, mientras que la distribución vertical de la comunidad macrobentónica responde tanto a las condiciones bióticas como a las ambientales.

PALABRAS CLAVE: estuario, aguas de transición, escalas espaciales, distribución vertical, Laguna de Rocha, Uruguay.

\section{Abstract}

Rocha Lagoon is Part of the chain of coastal lagoons extended along Uruguayan Atlantic coast, which continues through southern Brazil. It presents a complex ecological dynamic due to the marine-brackish gradient. The benthic macrofauna is an important component of these estuarine environments which is affected by its complex dynamics. In this work, we analysed how sand-tongues (sand accumulation perpendicular to the sandbar) affect the macrobenthic fauna. We stablished the relationship between macrobenthos and environmental variables on a vertical (different depths within the sediment) and horizontal (both sides of the sand-tongues) scales, as well as evaluating the influence of the distance to the marine intrusion. In all the sampling points, the environmental parameters (organic matter, total phosphorus, dissolved oxygen, chlorophyll- $a$ and phaeopigments) showed a vertical trend (decreasing towards the bottom), and the macrobenthos responded to these changes. The dominance of Heleobia australis, Nephtys fluviatilis and Heteromastus similis was observed in different sampling points and sediment depths. There is a spatial partition of resources reflected by these changes in dominance, while the vertical distribution of the macrobenthic community responds to both biotic and environmental conditions.

KEYWORDS: estuary, transitional waters, spatial scale, vertical distribution, Rocha Lagoon, Uruguay.

\section{INTRODUCCIÓN}

Los estuarios son cuerpos de agua costeros semicerrados donde el ingreso del agua de mar desde el océano abierto se diluye significativamente en el agua dulce proveniente del drenaje terrestre, pudiendo sostener especies eurihalinas durante parte o todo su ciclo de vida (Perillo, 1995). Entre los sistemas estuarinos, las lagunas costeras son cuerpos de agua someros y salobres, separados del ambiente costero por una barra de arena que se conecta de forma intermitente con el océano por medio de un canal (Kjerfve, 1994). Los ecosistemas de las lagunas costeras son ecológicamente complejos debido a que están periódicamente expuestos a perturbaciones ambientales extremas, como inundaciones por aportes fluviales o intrusiones marinas (Conde y Rodríguez-Gallego, 2002).

Como consecuencia del estrés físico que representan los cambios abruptos de salinidad, provocado por la apertura de la barra, solo las especies capaces de enfrentar tales condiciones hacen uso permanente de estos hábitats, hecho que 
condiciona la ocurrencia de las especies en esos ambientes (Sconfietti, et al., 2003). En los ambientes estuarino-lagunares, la macrofauna bentónica es un componente importante dada la fuerte interacción entre éstos y la columna de agua (Day, et al., 2012). Algunos autores sugieren que el gradiente marino-salobre y su variabilidad temporal podrían explicar la variación en la distribución de los organismos bentónicos (Attrill, 2002; Day, et al., 2012). Además, la fauna también responde al tipo de sedimento, contaminantes y enriquecimiento orgánico, a través de mecanismos indirectos o directos (Giménez, et al., 2014).

Las lagunas costeras uruguayas forman parte de una cadena que se extiende por la costa oceánica y se continúa con las lagunas costeras del sur de Brasil. La cuenca Atlántica uruguaya abarca 9266 km² e incluye las lagunas costeras más importantes del país. Entre ellas, se destaca la Laguna de Rocha por su importancia ecológica y social (Wells y Daborn, 1998).

La Laguna de Rocha pertenece al Sistema Nacional de Áreas Protegidas del Uruguay, fue declarada Parque Nacional Lacustre y Área de Uso Múltiple por el Decreto 260/977 (Uruguay, 1977) y es de suma importancia por su alta biodiversidad y productividad (Knoppers, 1994; Conde y Rodríguez-Gallego, 2002; Conde, et al., 2003).

Esta laguna presenta una barra arenosa que se abre de forma natural periódicamente cuando el nivel de agua dentro de la laguna es superior a 1,30 m (Sommaruga y Conde, 1990) y de manera artificial cuando se considera conveniente de acuerdo a un protocolo establecido en el plan de manejo del área protegida (Conde, et al., 2019).

La vegetación circundante está dominada por extensas praderas donde se realizan actividades agrícola/ganaderas y bañados, mientras que al sur hay un cordón dunar (Rodríguez-Gallego, 2010).

El macrobentos de la laguna de Rocha se caracteriza por su baja diversidad, su composición espacial y temporalmente uniforme, y una abundancia que varía estacionalmente (Cardezo, 1989). Dominan los crustáceos (40\%), principalmente ostrácodos, seguidos por los moluscos (35\%), en particular el gasterópodo Heleobia australis y el bivalvo Erodona mactroides, y, por último, los poliquetos (25\%), dominados por Nephtys fluviatilis (Arocena y Prat, 2006).

Se realizan actividades de pesca artesanal en dos pueblos aledaños, La Riviera y Puerto de los Botes, que concentran aproximadamente 49 personas asentadas de forma permanente. Los fines de semana, o según la época del año (producción pesquera y zafra del camarón), se pueden encontrar cientos de pescadores deportivos y familias que los visitan como lugar de descanso (Vitancurt y Fagetti, 1995).

Antiguamente, la laguna se comunicaba con el océano por otro sitio más al este de la actual apertura, denominado Barra Vieja (Panario y Gutiérrez, 2011). A causa del oleaje interno de la laguna (Conde, et al., 2015), se formaron allí acumulaciones de arena perpendiculares a la barra, comúnmente llamadas «lenguas de arena». Estos microambientes se caracterizan por sus diferencias ambientales de un lado y otro y por su distribución espacial, que determina mayor/menor distancia de la intrusión salina que ocurre en el sistema lagunar (Figura 1).

Estudios previos sobre bentos realizados en lagunas costeras de Uruguay (Giménez, et al., 2006; Giménez, et al., 2014; Meerhoff, et al., 2013) indican que los procesos 
de pequeña escala juegan un rol importante al definir los patrones espaciales de las comunidades macrobentónicas. Sin embargo, son escasas las investigaciones que examinan la partición vertical del espacio (i.e. profundidad) a nivel de microescala espacial, tanto del ambiente físicoquímico como de la distribución de la fauna bentónica (Rodríguez, 2016; Touhami, et al., 2018).

El objetivo de este trabajo fue analizar cómo afecta la presencia de estas «lenguas de arena» a la fauna macrobentónica, relacionándola con los parámetros ambientales a escala vertical (dentro del sedimento) y a escala horizontal (a un lado y al otro de las lenguas de arena). A su vez, se estudió si la distancia a la intrusión de agua marina tiene un efecto en estas estructuras sedimentarias y sus comunidades.

Como primera hipótesis se espera encontrar diferencias en la estructura de la comunidad a un lado y otro de la lengua de arena (escala horizontal), así como también en un gradiente de profundidad (escala vertical), según las características granulométricas del sedimento. Además, se predice que en la lengua más cercana a la intrusión de agua salada se observará una comunidad macrobentónica diferente a las presentes en las lenguas más alejadas.

\section{MATERIALES Y MÉTODOS}

\section{Área de estudio}

Este trabajo se realizó en la Laguna de Rocha $\left(34^{\circ} 40^{\prime} \mathrm{S}, 54^{\circ} 16^{\prime} \mathrm{W}\right)$ en setiembre de 2016 (invierno-primavera austral), sobre tres lenguas de arena, en adelante nuestras estaciones (L) (Figura 1). El día del muestreo la barra de arena de la laguna se encontraba abierta.

La laguna tiene una superficie de $72 \mathrm{~km}^{2}$, profundidad media de $0,6 \mathrm{~m}$, profundidad máxima de 1,4 m y el área de la cuenca es de $1312 \mathrm{~km}^{2}$ (Clara, et al., 1994). La salinidad varía entre 0,5 y 33, exhibiendo una marcada variación de salinidad en sentido norte-sur. Los sedimentos son principalmente arenosos, aunque hacia el norte de la laguna aumenta su contenido de limo y arcilla (Sommaruga y Conde, 1990). Sus principales afluentes son los arroyos Las Conchas y Rocha (Clara, et al., 1994). 


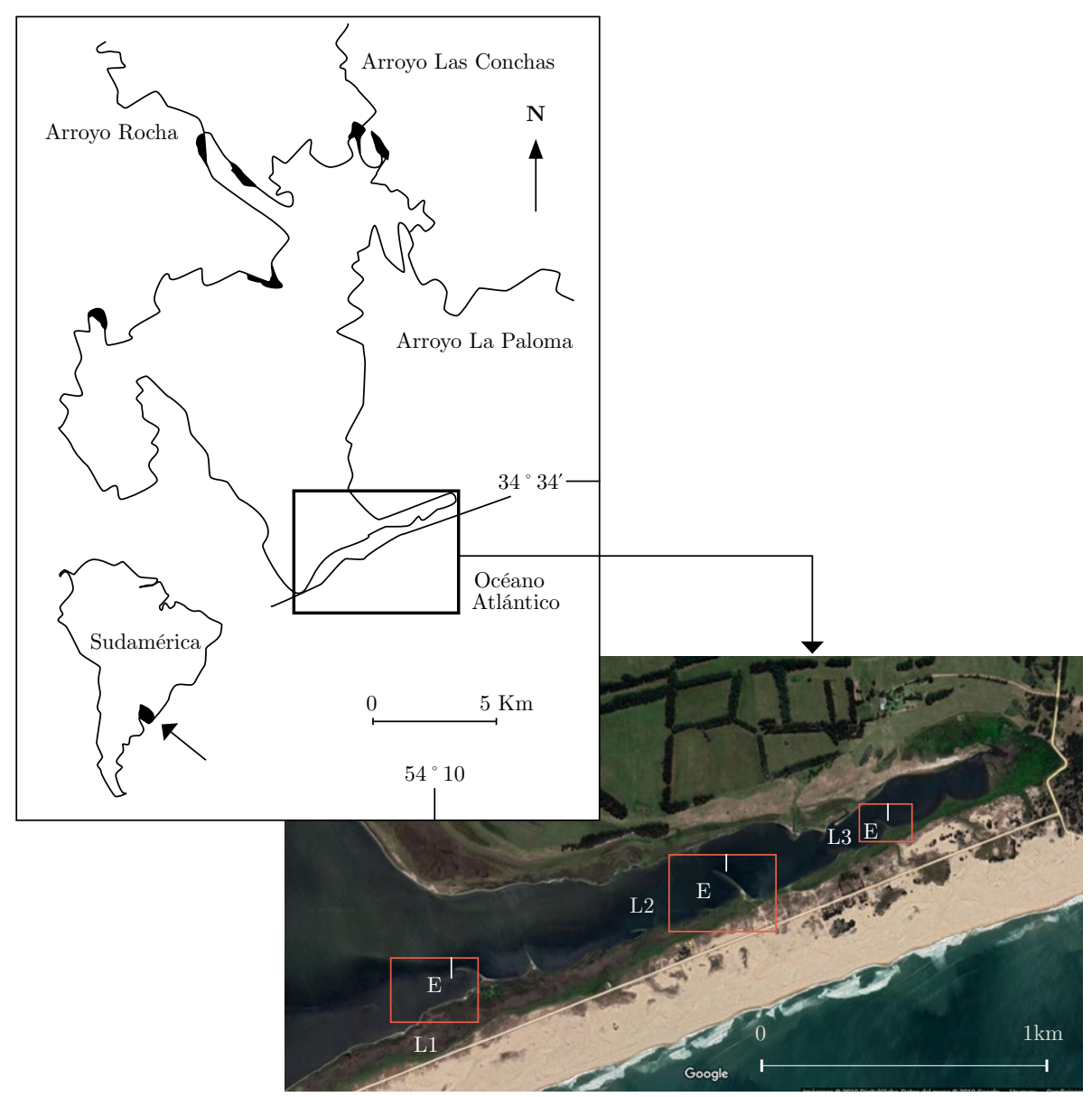

Figura 1. Mapa del área de estudio (modificado de Conde et al., 1999), seguido de la vista satelital de la barra vieja de la Laguna de Rocha. Se enmarcan en rojo las estaciones (Lenguas $=\mathrm{L}$ ) y los puntos de muestreo en cada estación $(\mathrm{E}=$ externo, $\mathrm{I}=$ interno $)$.

\section{Muestreo}

El muestreo fue realizado jerárquicamente y de forma anidado, sobre tres estaciones (L1, L2 y L3) y en dos puntos, externo (E) e interno (I) (Figura 1), que se dividieron en tres estratos: superficie, medio y fondo. Utilizando tubos de PVC de $12 \mathrm{~cm}$ de diámetro interno se extrajeron muestras de sedimento en cada punto, una para granulometría y tres para macrofauna, subdivididas en estratos de $3 \mathrm{~cm}$. Adicionalmente, con tubos de $\mathrm{PVC}(2,7 \mathrm{~cm}$ diámetro interno) se tomaron tres muestras (subdivididas en estratos: superficie, medio y fondo) para analizar fósforo total (Pt), materia orgánica (MO), clorofila- $a$ y feopigmentos. Un cuarto tubo fue utilizado para medir la concentración de oxígeno disuelto en el perfil de sedimento utilizando un sensor Unisense OX50. 


\section{Análisis de laboratorio}

Para determinar las características granulométricas se empleó el método de tamizado descripto en Suguio (1973). El Pt en sedimento se determinó siguiendo el método modificado de Valderrama (1981), el cual consiste en una digestión previa de las muestras para hidrolizar las formas fosforadas a fosfatos. Luego se determinaron los fosfatos con el método de Murphy y Riley (1962). Para MO se utilizó el procedimiento de pérdida de peso por ignición establecido en Byers et al. (1978). Utilizando el método de Lorenzen modificado por Sundbäck (1983) se determinaron concentraciones de clorofila- $a$ y feopigmentos. Por otro lado, las muestras destinadas a la identificación del macrobentos fueron tamizadas $(500 \mu \mathrm{m})$ en el campo, preservadas en alcohol $70 \%$ y posteriormente analizadas a través de lupa binocular (Olympus SZ61). Se observaron y contabilizaron por réplica y por estrato los organismos presentes, identificados en su gran mayoría a nivel específico utilizando claves de identificación regionales y ayuda de expertos (varios de los propios autores de este trabajo).

\section{Tratamiento de datos}

El tamaño medio de grano y su desviación estándar se determinaron por el método de Folk (1980) usando el programa GRADISTATv8 (Blott y Pye, 2001).

Los datos ambientales fueron normalizados y luego se realizó un análisis de componentes principales (PCA) para explorar la variación espacial en función de las estaciones/puntos/estratos. La matriz utilizada fue la de correlación y fue de seis variables (MO, Pt, oxígeno, clorofila- $a$, feopigmentos y porcentaje de fango más arena muy fina) por 18 muestras (tres estaciones, dos puntos, tres estratos).

Los datos de abundancia de las especies fueron utilizados para calcular la riqueza específica ( $\mathrm{S}$, número de taxa), $\mathrm{N}$ (número de individuos), J' (índice de equitatividad; Pielou, 1966) y H' (índice de Shannon-Wiener, con logaritmo en base e).

Se construyó una matriz de similaridad usando la transformación logarítmica $(\mathrm{x}+1)$ de la abundancia y el índice de Bray-Curtis (Bray y Curtis, 1957). Para observar la similaridad de la composición de comunidades macrobentónicas entre las distintas estaciones de muestreo y los distintos estratos se realizó un análisis de cluster (modo Q) y otro de ordenación (nMDS). También se realizó un análisis de similaridad de una vía (SIMPER, similaridad de porcentajes) para los parámetros abióticos y para las comunidades del macrobentos.

Todos los análisis fueron analizados mediante el software PRIMER 6.0 (Clarke y Gorley, 2006).

Asimismo, se realizaron ANOVAs, modelo jerárquico anidado, con S, N y H', en el caso de que se cumplieran los supuestos de homocedasticidad de varianza y normalidad (Prueba de Bartlett y Cochran). Las diferencias fueron evaluadas usando el Test de Tukey (contraste de medias) a posteriori (Zar, 1999), con un nivel de significancia del $95 \%$. En los casos en que las variables no cumplieron 
con los supuestos fueron transformadas por raíz cuadrada $\mathrm{y} / \mathrm{o} \log (\mathrm{x}+1)$. Estos análisis se realizaron utilizando el software PAST 3.23 (Hammer et al., 2001).

\section{RESULTADOS}

\section{Variables ambientales}

La fracción granulométrica dominante fue arena gruesa y las muestras fueron moderadamente bien seleccionadas, excepto L2I Superficie, que se clasificó como moderadamente seleccionada. El porcentaje de fango sumado a la arena muy fina presentó mayores valores en los puntos internos que en los externos (3-5\% vs. $<2 \%$, respectivamente). A su vez, se observó un marcado gradiente con mayores porcentajes en la superficie que descienden hacia los estratos inferiores, excepto en L2I donde el porcentaje en el fondo fue mayor que en el medio.

Los puntos internos se caracterizaron por tener mayores concentraciones de MO, clorofila- $a$ y Pt; esta tendencia ocurrió en los tres estratos. Las diferencias fueron evidenciadas con el ANOVA. El porcentaje de MO de L3 fue mayor a L1 en el estrato superficial, mientras que los estratos medio y fondo de L3I se diferenciaron del resto por presentar mayor porcentaje (Figura 2, Tabla 1).

La clorofila- $a$ mostró una concentración mayor en la superficie de L2I que en el resto de las muestras superficiales. En el estrato medio no se encontraron diferencias en la concentración de Clorofila- $a$ entre estaciones (lenguas). A su vez, esta fue mayor en L3I que en L3E, y en el estrato fondo de L3I fue mayor a todas las otras muestras de fondo (Figura 3, Tabla 1).

Verticalmente se observó una tendencia disminuyendo hacia el fondo de la concentración de MO, clorofila- $a$ y feopigmentos (Figura 2, 3).

TABLA 1. ANOVA de dos vías para evaluar las variaciones entre MO, Clo- $a$ y Pt en cada estrato y resultados del test pos hoc de Tukey. Donde: $\mathrm{CM}=$ Cuadrados medios, $\mathrm{Gl}=$ Grados de libertad, $\mathrm{MO}=$ porcentaje de materia orgánica total, Clo- $a=$ Clorofila- $a, \mathrm{Sup}=$ superficie, Med $=$ medio, Fon $=$ fondo.

\begin{tabular}{|c|c|c|c|c|c|c|c|c|}
\hline & \multirow{2}{*}{ Efecto } & \multirow{2}{*}{ SS } & \multirow{2}{*}{ GL } & \multirow{2}{*}{ CM } & \multirow{2}{*}{ F } & \multirow{2}{*}{$\mathbf{P}$} & \multicolumn{2}{|c|}{ Tukey } \\
\hline & & & & & & & CM & GL \\
\hline \multirow{3}{*}{ MO Sup } & $\mathrm{L}$ & 9,95 & 2 & 5,0 & 10,57 & $0,002^{*}$ & 0.47 & 12 \\
\hline & E O I & 15,34 & 1 & 15,3 & 32,59 & $0,000^{*}$ & 0,47 & 12 \\
\hline & $\mathrm{L}^{*} \mathrm{E} \mathrm{O} \mathrm{I}$ & 2,95 & 2 & 1,5 & 3,14 & 0,080 & 0,47 & 12 \\
\hline \multirow{3}{*}{ MO Med } & $\mathrm{L}$ & 6,79 & 2 & 3,4 & 38,92 & $0,000^{*}$ & 0,09 & 12 \\
\hline & E O I & 4,69 & 1 & 4,7 & 53,80 & $0,000^{*}$ & 0,09 & 12 \\
\hline & $\mathrm{L}^{*} \mathrm{E} \mathrm{O} \mathrm{I}$ & 4,96 & 2 & 2,5 & 28,44 & $0,000^{*}$ & 0,09 & 12 \\
\hline
\end{tabular}




\begin{tabular}{|c|c|c|c|c|c|c|c|c|}
\hline & \multirow{2}{*}{ Efecto } & \multirow{2}{*}{ SS } & \multirow{2}{*}{ GL } & \multirow{2}{*}{ CM } & \multirow{2}{*}{$\mathbf{F}$} & \multirow{2}{*}{$\mathbf{P}$} & \multicolumn{2}{|c|}{ Tukey } \\
\hline & & & & & & & CM & GL \\
\hline \multirow{3}{*}{ MO Fon } & $\mathrm{L}$ & 2,38 & 2 & 1,2 & 33,03 & $0,000^{*}$ & 0,04 & 12 \\
\hline & E O I & 0,91 & 1 & 0,9 & 25,18 & $0,000^{*}$ & 0,04 & 12 \\
\hline & $\mathrm{L}^{*} \mathrm{E} \mathrm{O} \mathrm{I}$ & 0,51 & 2 & 0,3 & 7,07 & $0,009^{*}$ & 0,04 & 12 \\
\hline \multirow{3}{*}{ Clo- $a$ Sup } & $\mathrm{L}$ & 241,00 & 2 & 120,5 & 8,29 & $0,005^{*}$ & 14,54 & 12 \\
\hline & E O I & 144,40 & 1 & 144,4 & 9,93 & $0,008^{*}$ & 14,54 & 12 \\
\hline & $\mathrm{L}^{*} \mathrm{E} O \mathrm{I}$ & 652,10 & 2 & 326,1 & 22,43 & $0,000^{*}$ & 14,54 & 12 \\
\hline \multirow{3}{*}{$\begin{array}{l}\text { Raíz Clo- } a \\
\text { Med }\end{array}$} & $\mathrm{L}$ & 2,99 & 2 & 1,5 & 3,86 & 0,051 & 0,39 & 12 \\
\hline & E O I & 4,06 & 1 & 4,1 & 10,48 & $0,007^{*}$ & 0,39 & 12 \\
\hline & $\mathrm{L}^{*} \mathrm{E} \mathrm{O} \mathrm{I}$ & 2,06 & 2 & 1,0 & 2,67 & 0,110 & 0,39 & 12 \\
\hline \multirow{3}{*}{ Clo- $a$ Fon } & $\mathrm{L}$ & 18,02 & 2 & 9,0 & 9,35 & $0,004^{*}$ & 0,96 & 12 \\
\hline & E O I & 5,38 & 1 & 5,4 & 5,58 & $0,036^{*}$ & 0,96 & 12 \\
\hline & $\mathrm{L}^{*} \mathrm{E} \mathrm{O} \mathrm{I}$ & 23,47 & 2 & 11,7 & 12,17 & $0,001^{*}$ & 0,96 & 12 \\
\hline \multirow{3}{*}{ Pt Sup } & $\mathrm{L}$ & 26200,00 & 2 & 13100,0 & 536,30 & $0,000^{*}$ & 24,41 & 12 \\
\hline & E O I & 16900,00 & 1 & 16900,0 & 693,70 & $0,000^{*}$ & 24,41 & 12 \\
\hline & $\mathrm{L}^{*} \mathrm{E} \mathrm{O} \mathrm{I}$ & 10100,00 & 2 & 5057,0 & 207,20 & $0,000^{*}$ & 24,41 & 12 \\
\hline \multirow{3}{*}{ Pt Med } & $\mathrm{L}$ & 7646,00 & 2 & 3823,0 & 37,11 & $0,000^{*}$ & 103,01 & 12 \\
\hline & E O I & 8734,00 & 1 & 8734,0 & 84,79 & $0,000^{*}$ & 103,01 & 12 \\
\hline & $\mathrm{L}^{*} \mathrm{E} \mathrm{O} \mathrm{I}$ & 4067,00 & 2 & 2034,0 & 19,74 & $0,000^{*}$ & 103,01 & 12 \\
\hline \multirow{3}{*}{ Pt Fon } & $\mathrm{L}$ & 189,90 & 2 & 95,0 & 1,12 & 0,358 & 84,83 & 12 \\
\hline & E O I & 432,70 & 1 & 432,7 & 5,10 & $0,043^{*}$ & 84,83 & 12 \\
\hline & $\mathrm{L}^{*} \mathrm{E}$ O I & 55,70 & 2 & 27,9 & 0,33 & 0,726 & 84,83 & 12 \\
\hline
\end{tabular}




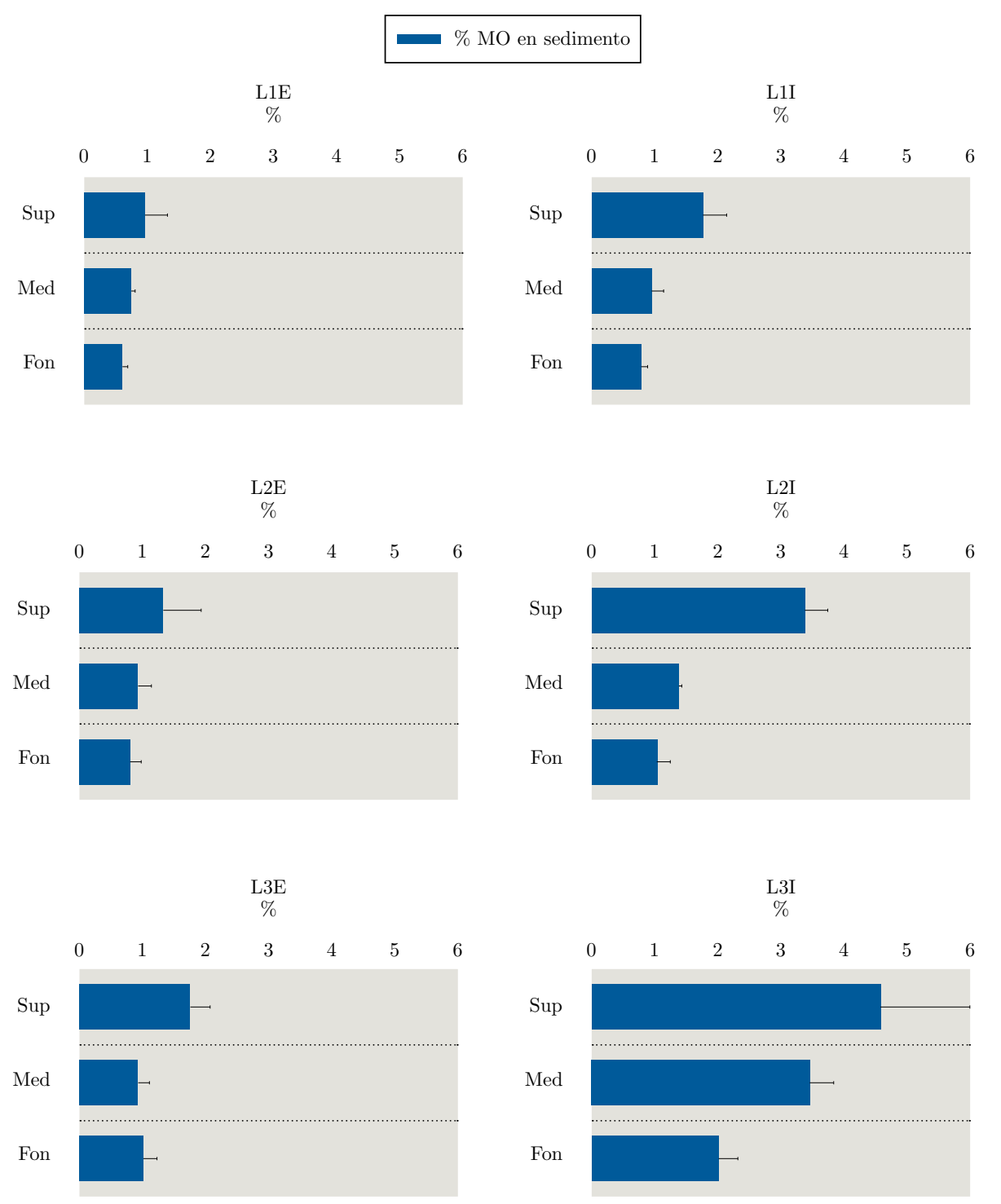

Figura 2. Porcentaje de materia orgánica en sedimento analizado por estratos ( Sup=superficie, Med=medio y Fon=fondo) en cada estación ( $\mathrm{L}=$ lengua) en sus dos puntos $(\mathrm{E}=$ externo e $\mathrm{I}=$ interno). 


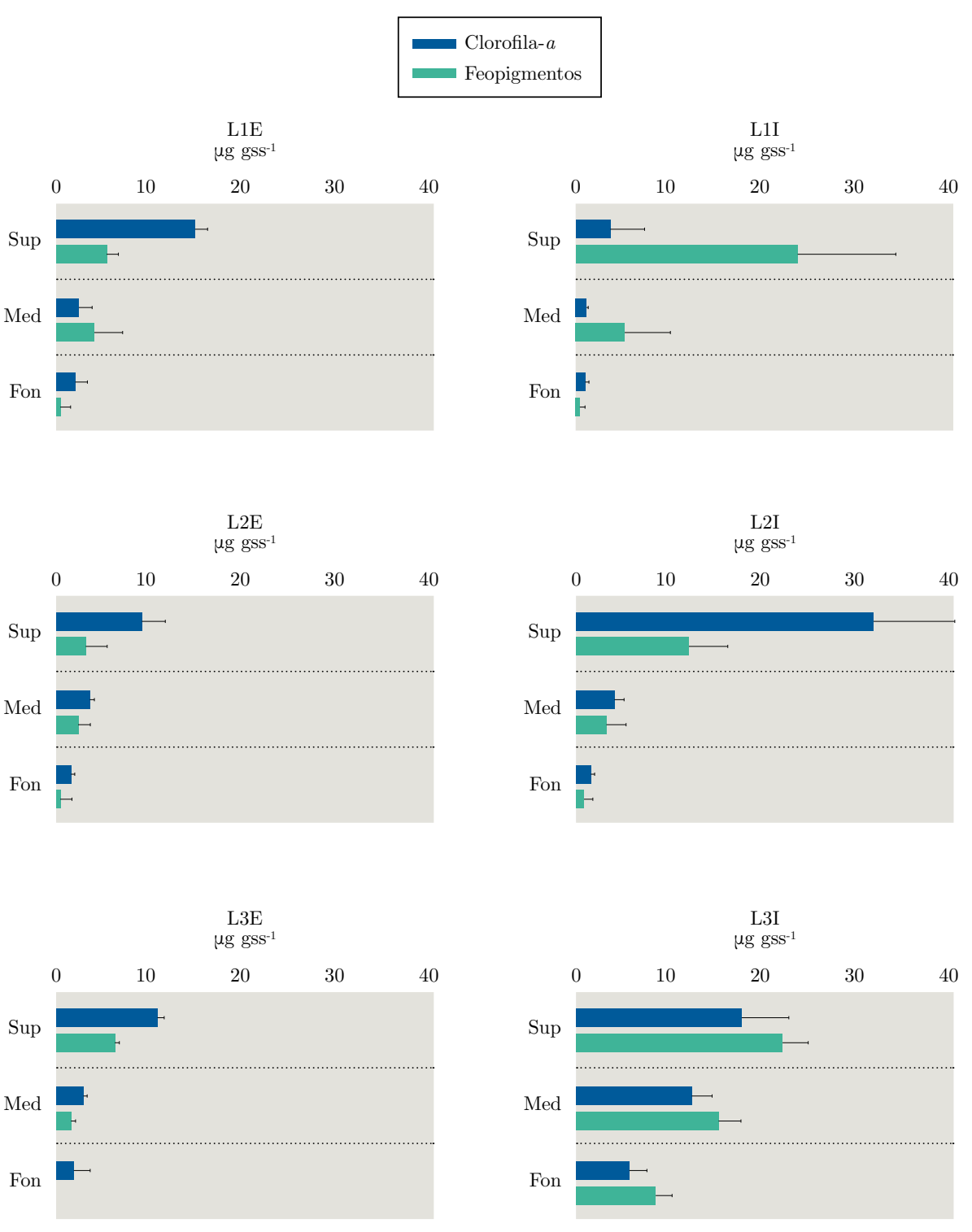

Figura 3. Concentración de clorofila- $a$ (en azul) y feopigmentos (en verde) en sedimento analizada por estratos (Sup=superficie, Med=medio y Fon=fondo) con sus respectivos desvíos estándar en cada estación ( $\mathrm{L}=$ lengua) en sus dos puntos $(\mathrm{E}=$ externo e $\mathrm{I}=$ interno).

El ANOVA para Pt mostró que L3I superficie fue mayor que el resto de las muestras. En medio L3I fue mayor a las demás muestras de este estrato. En el fondo los puntos internos presentaron mayores valores que los externos (Figura 4). 


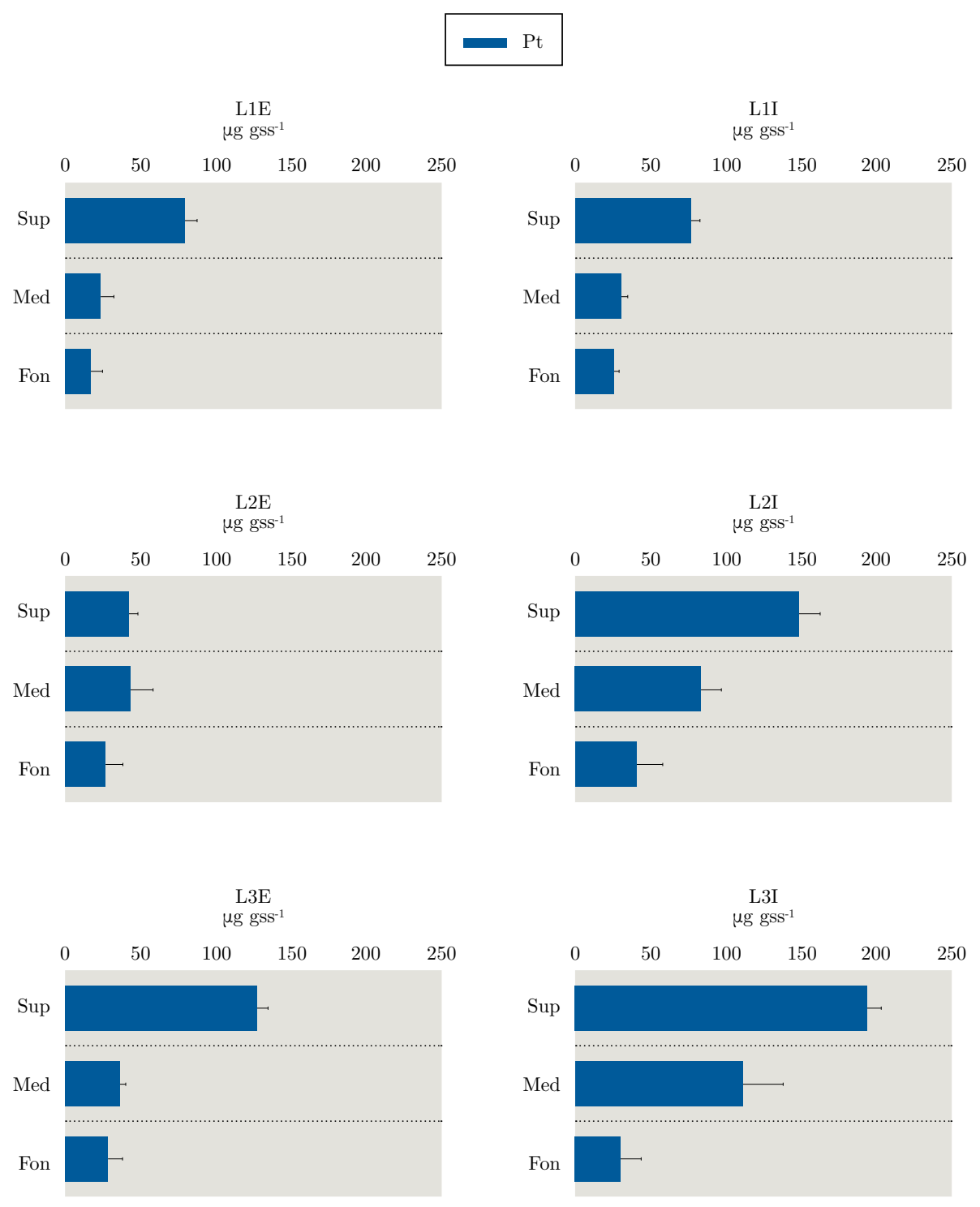

Figura 4. Concentración de Fósforo total (Pt) en sedimento ( $\mu \mathrm{g}$ gss $\left.^{-1}\right)$ analizada por estratos (Sup=superficie, Med=medio y Fon=fondo) con sus respectivos desvíos estándar en cada estación ( $\mathrm{L}=$ lengua) en sus dos puntos ( $\mathrm{E}=$ externo e $\mathrm{I}=$ interno).

En relación al oxígeno disuelto en el interior de la columna de sedimento se observó que los puntos internos presentaron menores concentraciones que externos, que a su vez disminuyó más acusadamente cuanto mayor fue la profundidad en ambos puntos (Figura 5). 


\section{- $\mathrm{O}_{2}$}

L1E

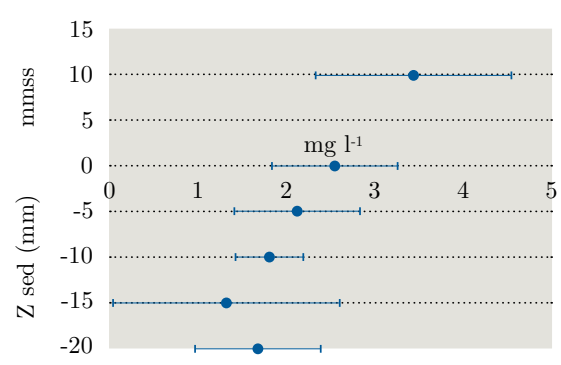

L2E

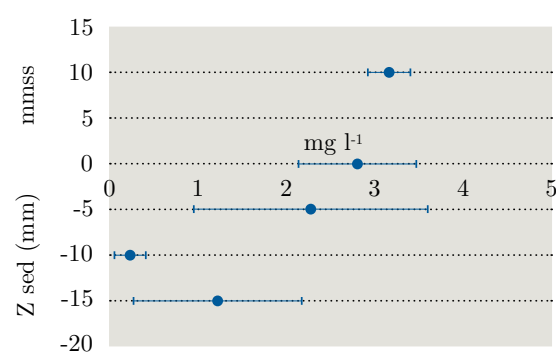

L3E

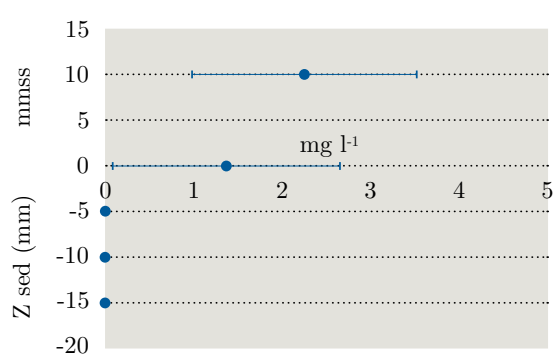

L1I

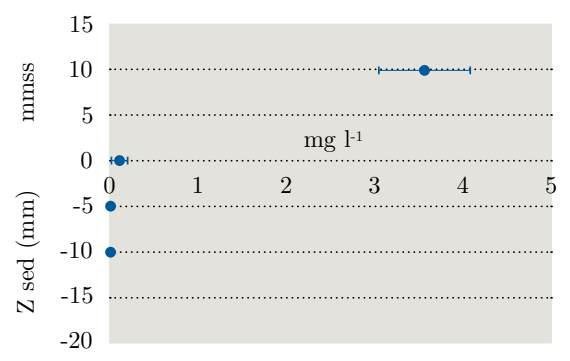

L2I

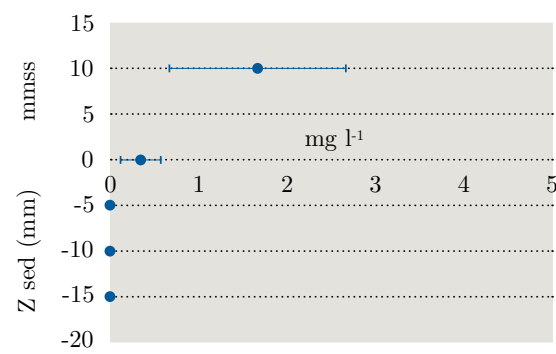

L3I

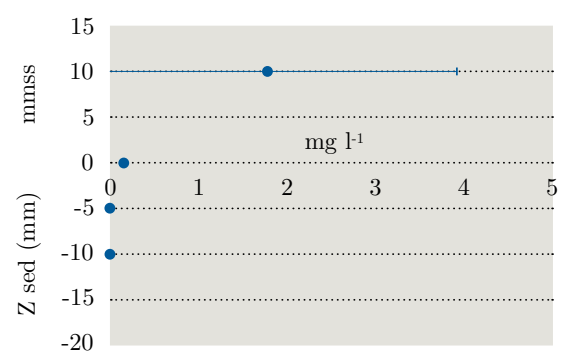

Figura 5. Concentración de oxígeno disuelto $\left(\mathrm{O}_{2}\right)$ en sedimento. Se representa en el eje de ordenadas la altura del agua (por encima del cero, mmss) y la profundidad del sedimento (valores negativos, z sed $(\mathrm{mm})$ ); y en el eje de abscisas la concentración $\left(\mathrm{mg} \mathrm{l}^{-1}\right)$. Cada valor de $\mathrm{O}_{2}$ se observa con su desviación estándar.

El resultado del PCA con las variables ambientales (Figura 6) explicó un 85\% de la varianza considerando los dos primeros ejes. El eje 1 se correlacionó positivamente con el porcentaje de MO, Pt, clorofila- $a$, feopigmentos y porcentaje de fango, explicando el $68 \%$ de la varianza, mientras que el eje 2 se correlacionó positivamente 
con el $\mathrm{O}_{2}$ en sedimento, explicando el $17 \%$ de la variación de los datos. Las muestras L2I superficial y L3I superficial se diferenciaron del resto por su mayor porcentaje de MO, Pt, clorofila- $a$, feopigmentos y porcentaje de fango. Las muestras de los estratos superficiales correspondientes a las estaciones L1E, L2E y L3E se diferenciaron del resto por sus altas concentraciones de $\mathrm{O}_{2}$. A su vez, se observa que las muestras de fondo fueron más similares entre sí desde el punto de vista ambiental, seguidas por las de medio y superficie.

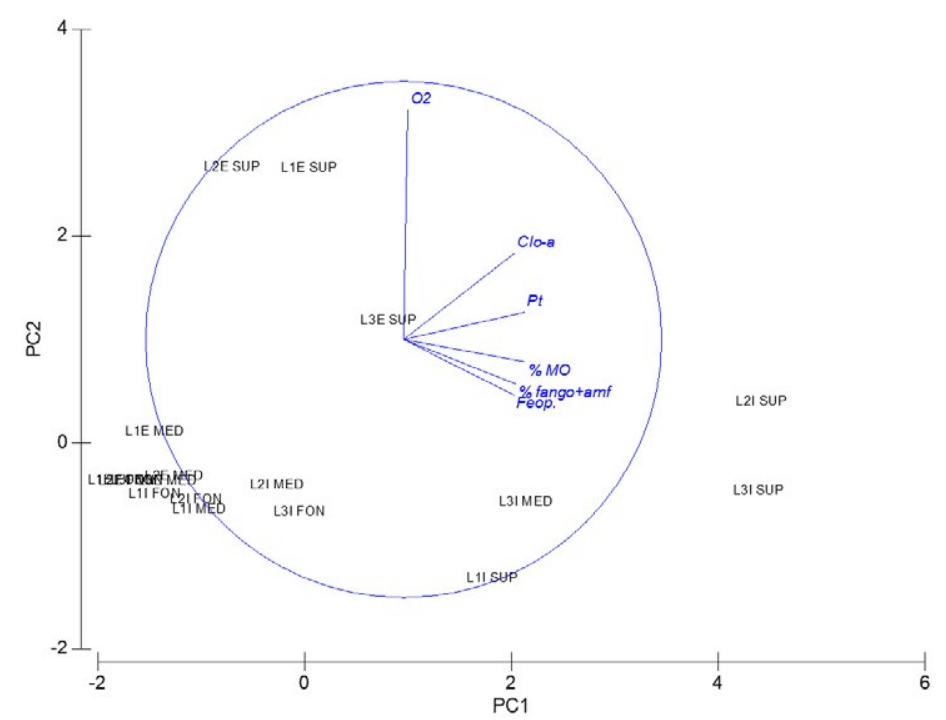

Figura 6. Análisis en componentes principales de las variables ambientales para los estratos de cada punto de muestreo (\%MO: porcentaje de materia orgánica, Pt: Fósforo total, Clo- $a$ : Clorofila- $a$, Feop: Feopigmentos, $\mathrm{O}_{2}$ : Oxígeno disuelto, $\%$ de fango + amf: porcentaje de fango más arena muy fina).

\section{Patrones macrofaunales}

Las tres estaciones presentaron una composición taxonómica similar (Tabla 2), la cual estuvo representada por 13 taxa, típicamente estuarinas. El pequeño gasterópodo Heleobia australis dominó en los estratos superficiales de L1, mientras que en las superficies de L2 y L3 predominó el poliqueto Nephtys fluviatilis. El poliqueto Heteromastus similis presentó mayor abundancia en los puntos internos que en los externos, aumentando desde L1 a L3 y dominando en los estratos medios. Los estratos superficiales se destacaron por presentar las mayores abundancias en todas las estaciones.

Los resultados de los ANOVA evidenciaron que la abundancia (N) superficial fue mayor en L1 que en L3; en el medio los puntos internos presentaron mayor $\mathrm{N}$ que los externos y en L3I fue mayor al resto de las muestras. No se observaron diferencias entre las muestras de fondo (Tabla 3). En cuanto a la S y H', no hubo 
diferencias en cada estrato analizado (Figura 7, Tabla 3). Los estratos superficiales de L2 y L3 presentaron alta J', mientras que los de L1 presentaron baja, debido principalmente a la dominancia de $H$. australis (Figura 7, Tabla 2).

TABLA 2. Abundancia (n. individuos $/ 340 \mathrm{~cm}^{3}$ ) de macrofauna en sedimento analizada por estratos ( $\mathrm{S}=$ superficie, $\mathrm{M}=$ medio y $\mathrm{F}=$ fondo) en cada estación $(\mathrm{L}=$ lengua) en sus dos puntos $(\mathrm{E}=$ externo, $\mathrm{I}=$ interno).

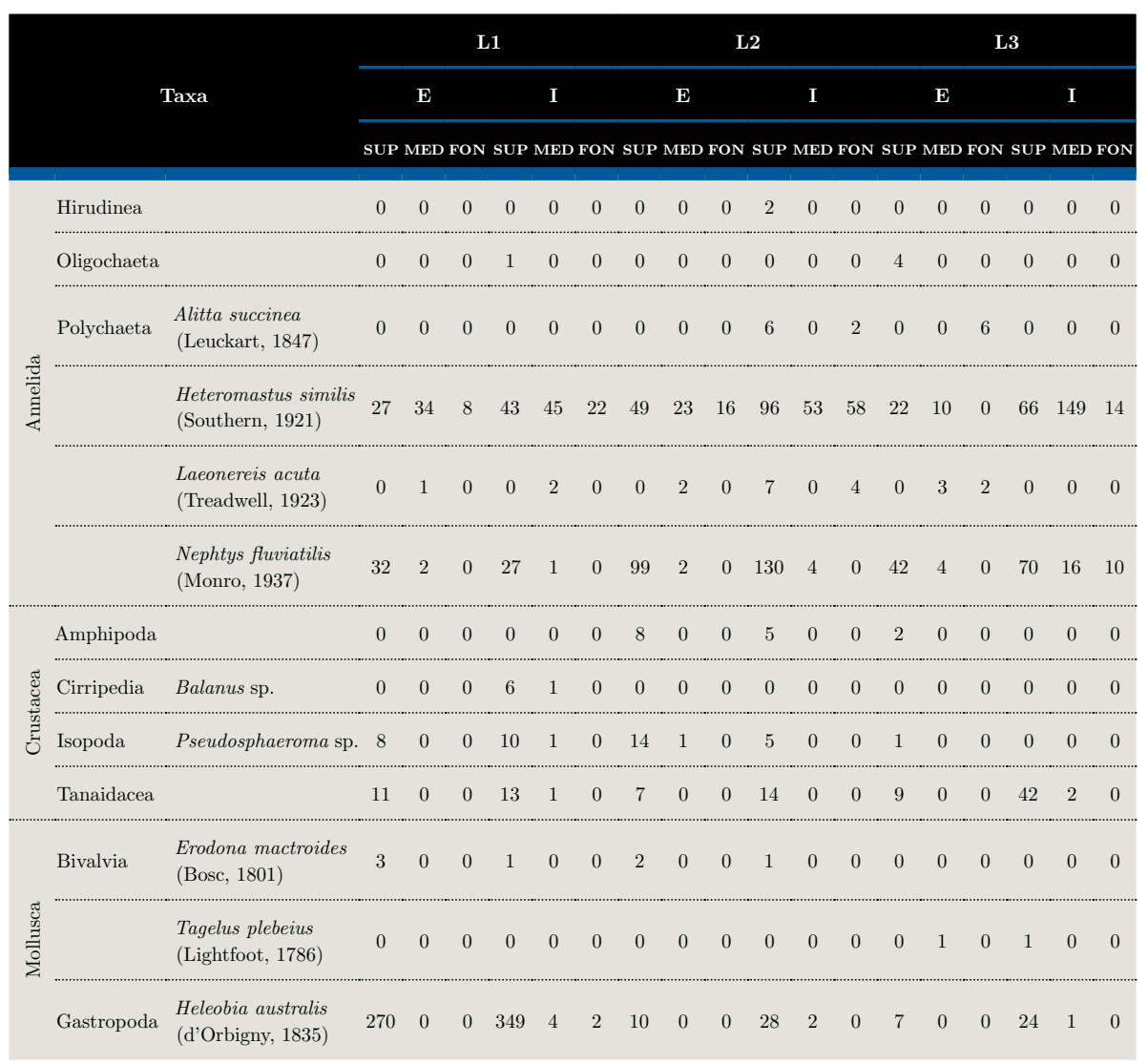




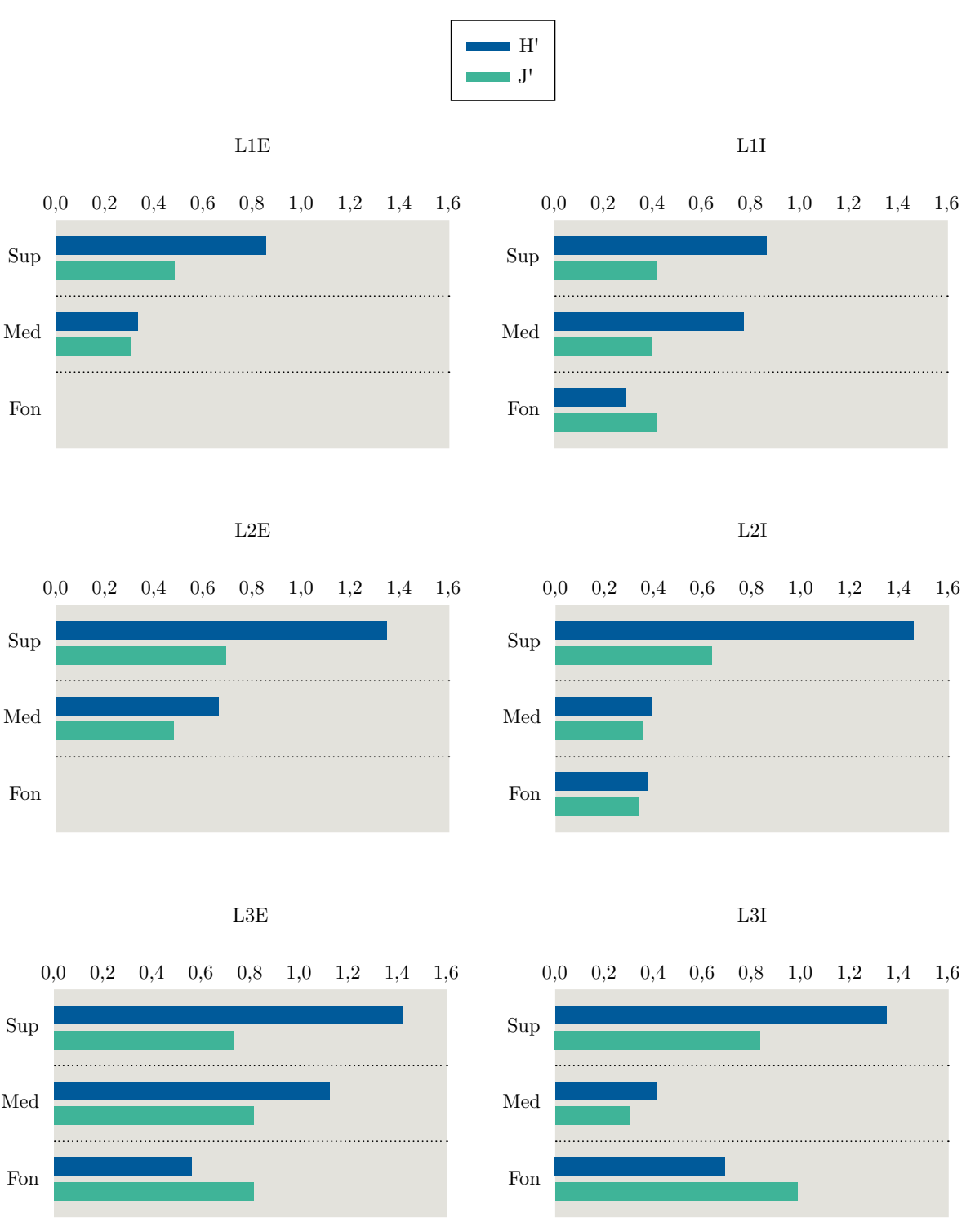

Figura 7. Índices de diversidad de Shannon (H’, en azul, bits/individuos) y equitatividad de Pielou (1966) ( $\mathrm{J}^{\prime}$, en verde) analizados por estratos (Sup=superficie, Med=medio y Fon=fondo) en cada estación ( $\mathrm{L}=$ lengua) en sus dos puntos $(\mathrm{E}=$ externo e $\mathrm{I}=$ interno). 
TABLA 3. ANOVA de dos vías para evaluar las variaciones entre $\mathrm{N}, \mathrm{S}$ y H' en cada estrato y resultados del test pos hoc de Tukey. Donde: $\mathrm{CM}=$ Cuadrados medios, $\mathrm{Gl}=$ Grados de libertad, $\mathrm{N}=$ Abundancia, $\mathrm{S}=$ Riqueza, H'= Diversidad de Shannon, Sup $=$ superficie, Med $=$ medio, Fon $=$ fondo.

\begin{tabular}{|c|c|c|c|c|c|c|c|c|}
\hline & \multirow{2}{*}{ Efecto } & \multirow{2}{*}{ SS } & \multirow{2}{*}{ GL } & \multirow{2}{*}{ CM } & \multirow{2}{*}{$\mathbf{F}$} & \multirow{2}{*}{$\mathbf{P}$} & \multicolumn{2}{|c|}{ Tukey } \\
\hline & & & & & & & CM & GL \\
\hline \multirow{3}{*}{ N Sup } & $\mathrm{L}$ & 22200,00 & 2 & 11100,00 & 6,769 & $0,011^{*}$ & 1638,40 & 12 \\
\hline & E O I & 5724,00 & 1 & 5724,00 & 3,494 & 0,086 & 1638,40 & 12 \\
\hline & $\mathrm{L}^{*} \mathrm{E} \mathrm{O} \mathrm{I}$ & 24,00 & 2 & 12,00 & 0,007 & 0,993 & 1638,40 & 12 \\
\hline \multirow{3}{*}{ N Med } & $\mathrm{L}$ & 1037,00 & 2 & 518,00 & 18,81 & $0,000^{*}$ & 27,56 & 12 \\
\hline & E O I & 2200,00 & 1 & 2200,00 & 79,84 & $0,000^{*}$ & 27,56 & 12 \\
\hline & L*E O I & 1764,00 & 2 & 882,00 & 32,01 & $0,000^{*}$ & 27,56 & 12 \\
\hline \multirow{3}{*}{$\mathrm{N} \log$ Fon } & $\mathrm{L}$ & 0,31 & 2 & 0,16 & 0,465 & 0,639 & 0,34 & 12 \\
\hline & E O I & 0,27 & 1 & 0,27 & 0,806 & 0,387 & 0,34 & 12 \\
\hline & $\mathrm{L}^{*} \mathrm{E} O \mathrm{I}$ & 0,00 & 2 & 0,00 & 0,005 & 0,995 & 0,34 & 12 \\
\hline \multirow{3}{*}{ S Sup } & $\mathrm{L}$ & 17,33 & 2 & 8,67 & 3,467 & 0,065 & 2,50 & 12 \\
\hline & E O I & 1,39 & 1 & 1,39 & 0,556 & 0,470 & 2,50 & 12 \\
\hline & $\mathrm{L}^{*} \mathrm{E}$ O I & 1,78 & 2 & 0,89 & 0,356 & 0,708 & 2,50 & 12 \\
\hline \multirow{3}{*}{ S Med } & $\mathrm{L}$ & 0,44 & 2 & 0,22 & 0,138 & 0,873 & 1,61 & 12 \\
\hline & E O I & 2,72 & 1 & 2,72 & 1,690 & 0,218 & 1,61 & 12 \\
\hline & L*E O I & 1,78 & 2 & 0,89 & 0,552 & 0,590 & 1,61 & 12 \\
\hline \multirow{3}{*}{ S Fon } & $\mathrm{L}$ & 0,78 & 2 & 0,39 & 0,412 & 0,671 & 0,94 & 12 \\
\hline & E O I & 0,50 & 1 & 0,50 & 0,529 & 0,481 & 0,94 & 12 \\
\hline & $\mathrm{L}^{*} \mathrm{E}$ O I & 0,33 & 2 & 0,17 & 0,176 & 0,840 & 0,94 & 12 \\
\hline \multirow{3}{*}{$\mathrm{H}^{\prime}$ Sup } & $\mathrm{L}$ & 0,50 & 2 & 0,25 & 2,980 & 0,089 & 0,08 & 12 \\
\hline & E O I & 0,02 & 1 & 0,02 & 0,257 & 0,621 & 0,08 & 12 \\
\hline & $\mathrm{L}^{*} \mathrm{E}$ O I & 0,00 & 2 & 0,00 & 0,002 & 0,998 & 0,08 & 12 \\
\hline \multirow{3}{*}{$\mathrm{H}^{\prime}$ Med } & $\mathrm{L}$ & 0,03 & 2 & 0,02 & 0,100 & 0,906 & 0,16 & 12 \\
\hline & E O I & 0,01 & 1 & 0,01 & 0,051 & 0,824 & 0,16 & 12 \\
\hline & $\mathrm{L}^{*} \mathrm{E}$ O I & 0,32 & 2 & 0,16 & 1,002 & 0,396 & 0,16 & 12 \\
\hline \multirow{3}{*}{$\mathrm{H}^{\prime}$ Fon } & $\mathrm{L}$ & 0,06 & 2 & 0,03 & 0,554 & 0,589 & 0,05 & 12 \\
\hline & E O I & 0,03 & 1 & 0,03 & 0,660 & 0,432 & 0,05 & 12 \\
\hline & $\mathrm{L}^{*} \mathrm{E}$ O I & 0,07 & 2 & 0,04 & 0,731 & 0,501 & 0,05 & 12 \\
\hline
\end{tabular}


Los resultados del análisis SIMPER (similaridad porcentual) mostraron una similaridad del $73 \%$ entre el ensamble del estrato de superficie, fundamentalmente debido a la influencia de los poliquetos $N$. fluviatilis y $H$. similis. En el estrato medio y de fondo también la similaridad (68\% y 45\%, respectivamente) ocurrió por el poliqueto depositívoro $H$. similis. A su vez, el porcentaje de disimilaridad entre los ensambles de superficie y medio fue de 54 , donde $H$. australis y $N$. fluviatilis fueron las especies que más contribuyeron. La disimilaridad entre los ensambles de superficie y fondo fue del $73 \%$, con una contribución por igual de $N$. fluviatilis y H. australis. Los ensambles de fondo y medio presentaron un $49 \%$ de disimilaridad, influenciada por $H$. similis y $N$. fluviatilis. Por lo tanto, las muestras de superficie son las más parecidas biológicamente, seguidas por las de medio y luego las de fondo.

El análisis nMDS mostró que la macrofauna de las 18 muestras presentó bajo nivel de similitud, lo cual se debió, principalmente, a que L3E fondo presentó atributos biológicos muy distintos al resto de las muestras (Figura 8). Esto se debió en particular a la ausencia de $H$. similis en el estrato de fondo de L3E. A pesar de ello, se observaron claramente agrupadas entre sí las muestras de superficie, medio y fondo, a excepción de L3E fondo y L2I fondo. A su vez, las muestras de fondo se encontraron más cercanas a las de medio que a las de superficie, al igual que en el análisis SIMPER.

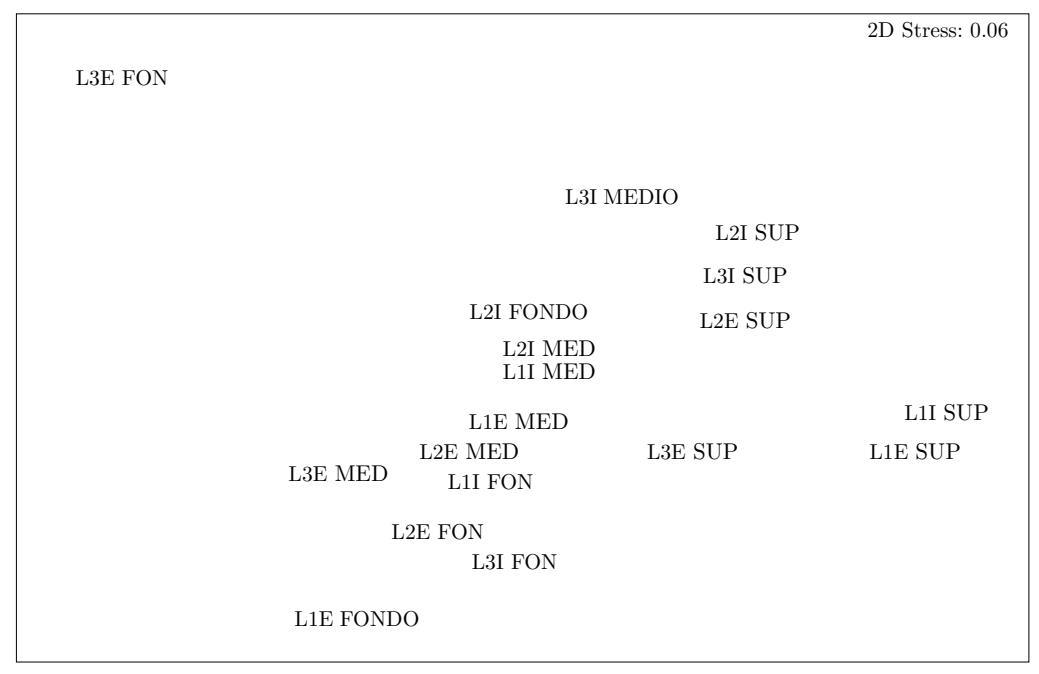

Figura 8. Escalamiento multidimensional no métrico nMDS. Abreviaturas: L1, L2, L3=estaciones (lenguas); E, I=externo e interno respectivamente; $\mathrm{SUP}=$ Superficie, $\mathrm{MED}=$ Medio, $\mathrm{FON}=$ Fondo.

\section{Discusión}

En este trabajo se demostró que existe una variación en la distribución vertical y horizontal de pequeña y microescala en la estructura de las comunidades macrobentónicas en la laguna de Rocha. Las diferencias ambientales observadas entre las estaciones fueron determinantes en la explicación de los patrones de composición y abundancia 
del macrobentos. Sin embargo, no se encontraron diferencias en la estructura de los ensambles macrobentónicos a un lado y al otro de las distintas estaciones (LE x LI). Se observaron patrones de distribución del macrobentos atendiendo a un gradiente entre las estaciones y de profundidad dentro del sedimento, que se relaciona con los parámetros ambientales obtenidos. Estudios previos mostraron este comportamiento y destacaron la importancia del estrato superficial en cuanto a la abundancia macrobentónica debido a su rol fundamental en la transferencia energética entre el sedimento y la columna de agua (Venturini, et al., 2011; Touhami, et al., 2018).

Los puntos internos y más alejados de la intrusión marina presentaron una hidrodinámica baja que favorece la deposición de sedimentos finos y facilita la acumulación de materia orgánica (Venturini, et al., 2004). Esta materia orgánica acumulada es degradada por parte de bacterias que consumen oxígeno (Muniz, et al., 2013). El porcentaje medio de MO (valores $<6 \%$ ) coincidió con lo obtenido en trabajos previos (Sommaruga y Conde, 1990; Rodríguez-Gallego, 2010; Meerhoff, et al., 2013). Los mayores porcentajes de MO en las estaciones (lenguas) más cercanas a la parte continental posiblemente se relacionan al aporte de desechos urbanos filtrados y conducidos hacia la laguna por las aguas que allí confluyen (Rodríguez-Gallego, 2010).

La clorofila- $a$ es un indicador indirecto de la productividad primaria del ambiente y es utilizada como una adecuada partícula traza debido a que indica la rápida reacción de la macrofauna a los cambios en las condiciones ambientales. La abundancia de organismos y la concentración de clorofila- $a$ presentaron una alta correlación, esto puede deberse al rol de los organismos como generadores de patrones de bioturbación (Morys, et al., 2017). Las concentraciones de clorofila- $a$ fueron, en promedio, menores a las analizadas por Rodríguez-Gallego (2010) para el conjunto de lagunas costeras. La mayor concentración de feopigmentos registrada en los puntos internos indicó el dominio de detritos orgánicos con algún grado de degradación (Fabiano y Danovaro, 1994). A su vez, los valores de fósforo se encontraron dentro del rango de valores observados en el trabajo de Rodríguez-Gallego (2010).

La distribución vertical del macrobentos en los sedimentos marinos puede cambiar a medida que la comunidad responde a la disponibilidad de oxígeno disuelto (Pearson y Rosenberg, 1978). Cuanto mayor fue la abundancia de organismos, mayores fueron las concentraciones de $\mathrm{O}_{2}$ observadas. La primera respuesta del macrobentos relacionada con el ecosistema a la disminución de las concentraciones de oxígeno implica una disminución de la bioturbación debido al cese de la alimentación y la excavación (Shivarudrappa, et al., 2019).

En cuanto a la comunidad de macroinvertebrados bentónicos presente, los resultados fueron acordes a los patrones generales encontrados en la laguna en estudios previos (Giménez, et al., 2006; Meerhoff, et al., 2013), donde la diversidad no fue alta y se observó la presencia de especies dominantes, como el gasterópodo Heleobia australis y los poliquetos Nephtys fluviatilis y Heteromastus similis.

Se observó una variación horizontal en la estructura de la comunidad entre las distintas estaciones (lenguas). En la lengua más cercana a la intrusión de agua marina la dominancia del gasterópodo epibentónico $H$. australis, especie oportunista de segundo orden (Muniz, et al., 2005), podría deberse a los disturbios 
provocados por la apertura de la barra que permite la dominancia de esta especie por recolonización rápida. Este proceso fue observado por Branco et al. (2007) en la laguna Imboassica en Brasil y por Rodríguez-Gallego (2010) en la Laguna de Rocha. Estudios previos, desarrollados en toda el área de la laguna, indican que organismos suspensívoros como Erodona mactroides y el depositívoro de superficie $H$. australis tienen densidades máximas limitadas a sedimentos con bajo contenido de fango y materia orgánica (Giménez, et al., 2006).

H. similis es un depositívoro subsuperficial $(<15 \mathrm{~cm})$ que se alimenta de materia orgánica (Bemvenuti, 1988), y junto con otro depositívoro infaunal, Laeonereis acuta (baja abundancia en este estudio), se destacó en estudios previos, en sedimentos con bajo o moderado contenido de fango y niveles intermedios de arena fina y media (Giménez, et al., 2014), lo cual se correlacionó con el patrón encontrado en los estratos de medio y fondo. Este patrón no fue observado en superficie debido a la presencia de $N$. fluviatilis, que podría predar sobre H. similis (Bemvenuti, 1994). La mayor presencia de $H$. similis en los puntos internos pudo deberse al mayor contenido orgánico en estos, ya que es una especie indicadora de enriquecimiento orgánico (Raz-Guzmán, 2000). Por otro lado, N. fluviatilis muestra un patrón mixto, y se destaca en sitios con bajo contenido de fango pero es común también en sitios fangosos (Giménez, et al., 2006). A su vez, al ser una especie detritívora, de amplia distribución gracias a sus larvas planctónicas, se la encontró asociada a mayores concentraciones de materia orgánica tanto en este trabajo como en anteriores (Arocena y Prat, 2006).

La distribución vertical de la comunidad macrobentónica responde tanto a las condiciones bióticas como a las ambientales. Como estrategia para particionar recursos (e.g. espacio) a microescala y para evitar la depredación, el estrés físico y/o interacciones competitivas por espacio o alimento, la macrofauna utilizaría el enterramiento diferencial (Rodríguez, 2016; Poznańska-Kakareko, et al., 2017). La característica de los organismos de homogeneizar el ambiente (Flach y Heip, 1996; Morys, et al., 2017), así como la competencia entre estos y la mayor compactación del sedimento a medida que aumenta la profundidad, podría explicar que las diferencias ambientales entre los estratos de medio y fondo fueran menores que las biológicas.

Este estudio aportó un abordaje a microescala espacial, que analizó y cuantificó la variación en profundidad de descriptores físicos y comunitarios a lo largo del eje transversal de la Barra Vieja, microambiente particular en el sistema lagunar estudiado. En esta oportunidad la comunidad bentónica se distribuyó verticalmente de acuerdo a las condiciones ambientales reinantes, que condicionan la presencia de las especies presentes.

\section{RECONOCIMIENTOS}

A la Facultad de Ciencias y al curso de Oceanografía Biológica II por haber brindado la oportunidad de realizar la investigación. También agradecer a los revisores y a la editora por las correcciones y sugerencias realizadas que contribuyeron a enriquecer este trabajo. NV y PM agradecen a SNI-ANII. 


\section{REFERENCIAS}

Arocena, R. y Prat, N., 2006. Efectos de la fertilización en el zoobentos de una laguna costera: experimentos con mesocosmos. En: Tundisi, J.G., Tundisi, T.M. y Galli, C.S., eds. Eutrofização na América do Sul: causas, consequências e tecnologías para gerenciamento e control. São Carlos: IIE. pp.387-412.

Attrill, M., 2002. A testable linear model for diversity trends in estuarios. En: Journal of Animal Ecology, 71, pp.262-269.

Bemvenuti, C.E, 1988. Impacto da predacao sobre Heteromastus similis Southern 1921 e Nephtys fluviatilis Monro 1937 (Annelida: Polychaeta) em fundos moles estuarinos. En: Atlántica, Rio Grande, 10(1), pp.85-102.

Bemvenuti, C.E., 1994. O Poliqueta Nephtys fluviatilis Monro, 1937, como predador da infauna na comunidade de fundos moles. En: Atlántica, 16, pp.87-98.

Blott, S.J. y Pye, K., 2001. Gradistat: a grain size distribution and statistics package for the analysis of unconsolidated sediments. En: Earth Surface Processes and Landforms, 26, pp.1237-1248.

Byers, S.C., Mills, E.L. y Stewart, P.L., 1978. A comparison of methods to determining organic carbon in marine sediments, with suggestion for a standard method. En: Hydrobiology, 58, pp.37-43.

Branco, C.W.C., Kozlowsky-Suzuki, B. y Esteves, F.A., 2007. Environmental changes and zooplancton temporal and spatial variation in a disturbed brazilian coastal lagoon. En: Brazilian Journal of Biology, 67(2), pp.251-262.

Bray, J.R. y Curtis, J.T., 1957. An ordination of the upland forest communities of southern Wisconsin. En: Ecological Monographs, 27, pp.325-349.

Cardezo, M.J., 1989. Caracterización de la fauna macrobentónica de la Laguna de Rocha (Uruguay). Montevideo: Facultad de Humanidades y Ciencias - UdelaR. (Tesis de grado).

Clara, M., Faggetti, C., Muzio, J., Picasso, G., Rudolf, J.C. y Vitancurt, J., 1994. Parque nacional Lacustre Laguna de Rocha. Una zona a proteger. Rocha: PROBIDES.

Clarke, K.R. y Gorley, R.N., 2006. PRIMER v6 user manual/tutorial. Plymouth: PRIMER-E.

Conde, D., Bonilla, S., Aubriot, L., de León, R. y Pintos, W., 1999. Comparison of the areal amount of chlorophyll $a$ of planktonic and attached microalgae in a shallow coastal lagoon. En: Hydrobiologia, 408/409, pp.285-291.

Conde, D. y Rodríguez-Gallego, L., 2002. Problemática ambiental y gestión de las lagunas costeras atlánticas de Uruguay. En: Domínguez, A., Prieto, R. y Achkar, M., eds. Perfil ambiental del Uruguay. Montevideo: Nordan-Comunidad. pp.149-166.

Conde, D., Rodríguez-Gallego, L. y Rodríguez-Graña, L., 2003. Análisis conceptual de las interacciones abióticas y biológicas entre el océano y las lagunas de la costa atlántica de Uruguay. Informe final PNUD/GEF/RLA/99/G31. Montevideo: Facultad de Ciencias, Sección Limnología. (FREPLATA-Ciencias, 8).

Conde, D., Vitancurt, J., Rodríguez-Gallego, L., de Álava, D., Verrastro, N., Chreties, C., Solari, S., Teixeira, L., Lagos, X., Piñeiro, G., Seijo, L., Caymaris, H. y Panario D., 2015. Solutions for sustainable coastal lagoon management: from conflict to 
the implementation of a consensual decision tree for artificial opening. En: Baztan, Juan, Chouinard, Omer, Jorgensen, Bethany, Tett, Paul, Vanderlinden, Jean-Paul y Vasseur, Liette, eds. Coastal zones: solutions for the 21st Century. pp.217-250.

Conde, D., Solari, S., de Álava, D., Rodríguez-Gallego, L., Verrastro, N., Chreties, C., Lagos, X., Piñeiro, G., Teixeira, L., Seijo, L., Vitancurt, J., Caymaris, H. y Panario, D., 2019. Ecological and social basis for the development of a sand barrier breaching model in Laguna de Rocha, Uruguay. En: Estuarine, Coastal and Shelf Science, 219, pp.300-316.

Day, J.W., Crump, B.C., Kemp, W.M. y Yáñez-Arancibia, A., 2012. Estuarine ecology. 2a ed. HobokEn: John Wiley \& Sons.

Fabiano, M. y Danovaro, R., 1994. Composition of organic matter in sediments facing a river estuary (Tyrrhenian Sea): relationships with bacteria and microphytobenthic biomass. En: Hydrobiologia, 277, pp.71-84.

Flach, E. y Heip, C., 1996. Vertical distribution of macrozoobenthos within the sediment of the continental slope of the Goban Spur area (NE Atlantic).

En: Marine Ecology Progress Series, 141, pp.55-66.

Folk, R., 1980. Petrology of sedimentary rocks. Texas: Hemphil Publishing Company.

Giménez, L., Dimitriadis, C., Carranza, A., Borthagaray, A. y Rodríguez, M., 2006. Unravelling the complex structure of a benthic community: a multiscalemultianalytical approach to an estuarine sandflat. En: Estuarine, Coastal and Shelf Science, 68, pp.462-472.

Giménez, L., Venturini, N., Kandratavicius, N., Huttonb, M., Lanfranconi, A., Rodríguez, M., Brugnoli, E. y Muniz, P., 2014. Macrofaunal patterns and animalsediment relationships in Uruguayan estuaries and coastal lagoons (Atlantic coast of South America). En: Journal of Sea Research, 87, pp.46-55.

Hammer, O., Harper, D.A.T. y Ryan, P.D., 2001. PAST: Paleontological Statistic software package for education and data analysis. Paleontologia electrónica, 4(1), pp.9.

Kjerfve, B., 1994. Coastal lagoon. En: Kjerfve, B., ed. Coastal lagoon processes. Amsterdam: Elsevier. (Oceanography Series, 60), pp.1-8.

Knoppers, B., 1994. Aquatic primary production in coastal lagoons. En: Kjerfve, B., ed. Coastal lagoon processes. Amsterdam: Elsevier. (Oceanography Series, 60), pp.243-285.

Meerhoff, E., Rodríguez-Gallego, L., Giménez, L., Muniz, P. y Conde, D., 2013. Spatial patterns of macrofaunal community structure in coastal lagoons of Uruguay. En: Marine Ecology Progress Series, 492, pp.97-110.

Morys, C., Powilleit, M. y Forster, S., 2017. Bioturbation in relation to the depth distribution of macrozoobenthos in the southwestern Baltic Sea. En: Marine Ecology Progress Series, 579, pp.19-36.

Muniz, P., Venturini, N., Pires-Vanin, A., Tommasi, L.R. y Borga, A., 2005. Testing the applicability of a Marine Biotic Index (AMBI) to assessing the ecological quality of soft-bottom benthic communities, in the South America Atlantic region. En: Marine Pollution Bulletin, 50, pp.624-637.

Muniz, P., Pires-Vanin, A. y Venturini, N., 2013. Vertical distribution patterns of macrofauna in a subtropical near-shore coastal area affected by urban sewage. En: Marine Ecology, 34, pp.233-250. 
Murphy, J. y Riley, J.P., 1962. A modified single solution method for the determination of phosphate in natural waters. En: Analytica Chimica Acta, 27, pp.31-36.

Panario, D. y Gutiérrez, O., 2011. Introducción a la geomorfología de lagunas costeras, lagos someros y charcas de Uruguay. En: García Rodríguez, F., ed. El holoceno en la zona costera de Uruguay. Montevideo: Universidad de la República, pp.49-64.

Pearson, T.N. y Rosenberg, R., 1978. Macrobenthic succession in relation to organic enrichment and pollution of the marine environment. En: Oceanogr. Mar. Biol. Annu. Rev, 16, pp.229-311.

Perillo G. M., 1995. Definitions and geomorphologic classifications of estuaries.

En: Developments in Sedimentology, 53, pp.17-47.

Pielou, E.C., 1966. The measurement of diversity in different types of biological collections. En: Journal of Theoretical Biology, 13, pp.131-144.

Poznańska-Kakareko, M., Budka, M., Żbikowski, J., Czarnecka, M., Kakareko, T., Jermacz, L. y Kobak, J., 2017. Survival and vertical distribution of macroinvertebrates during emersion of sandy substratum in outdoor mesocosmes. En: Fundamental and Applied Limnology, 190(1), pp.29-47

Raz-Guzmán, A., 2000. Crustáceos y poliquetos. En: de la Lanza-Espino, G., Pulido, S. H. y Pérez, J.L.C. Organismos indicadores de la calidad del agua y de la contaminación (bioindicadores). México: Plaza y Valdés. México. pp.265-308.

Rodríguez-Gallego, L., 2010. Eutrofización de las lagunas costeras de Uruguay: impacto y optimización de los usos del suelo. Montevideo: Facultad de Ciencias UdelaR. (Tesis de Doctorado).

Rodríguez, P., 2016. La tercera dimensión en ecología de playas arenosas: distribución vertical de macrofauna y factores abióticos en una playa oceánica de Uruguay. Montevideo: Facultad de Ciencias - UdelaR. (Tesis de grado).

Sconfietti, R., Marchini, A., Ambrogi, A.O. y Sacchi, C.F., 2003. The sessile benthic community patterns on hard bottoms in response to continental vs. marine influence in northern Adriatic lagoons. En: Oceanologica Acta, 26, pp.47-56.

Shivarudrappa, S., Rakocinski, C. y Briggs, K., 2019. Vertical distribution of macrobenthos in hypoxia-affected sediments of the northern Gulf of Mexico: applying functional metrics. En: Estuaries and Coasts, 42(9), pp.250-263. https:// doi.org/10.1007/s12237-018-0446-Z

Sommaruga, R. y Conde, D., 1990. Distribución de materia orgánica en los sedimentos recientes de la laguna de Rocha (Rocha, Uruguay). En: Atlântica, 12(1), pp.35-44.

Suguio, K., 1973. Introdução a sedimentologia. São Paulo: Edgard Blucher.

Sundbäck, K., 1983. Microphytobenthos on sand in shallow brackish water, Öresund Sweden. Primary production, chlorophyll a content and species composition (Diatoms) in relation to some ecological factors. Lund: University of Lund (Tesis de $\mathrm{PhD}$ ).

Touhami, F., Bazairi, H., Badaoui, B. y Benhoussa, A., 2018. Vertical distribution of benthic macrofauna in intertidal habitats frequented by shorebirds at Merja Zerga lagoon. En: Thalassas: An International Journal of Marine Sciences, 34(2), pp.255-265. 
Uruguay. Decreto 260/977, de 11 de mayo de 1977. Diario Oficial, 26 de mayo de 1977, p.911.

Valderrama, J.C., 1981. The simultaneous analysis of total $\mathrm{N}$ and total $\mathrm{P}$ in natural waters. En: Marine Chemistry, 10, pp.109-122.

Venturini, N., Tommasi, L.R., Bícego, M.C. y Martins, C.C., 2004. Characterisation of the benthic environment of a coastal area adjacent to an oil refinery, Todos os Santos bay (NE-Brazil). En: Brazilian Journal of Oceanography, 52, pp.123-134.

Venturini, N., Pires-Vanin, A.M.S., Salhi, M., Bessonart, M. y Muniz, P. 2011.

Polychaete response to fresh food supply at organically enriched coastal sites:Repercussion on bio turbation potential and trophic structure. En: Journal of Marine Systems, 88, pp.526-541.

Vitancurt, J. y Faggetti, C., 1995. Comunidades de pescadores del departamento de Rocha. Informe diagnóstico, recomendaciones y avances para un proyecto de desarrollo. Rocha: PROBIDES. (Documentos de Trabajo, 1).

Wells, P.G. y Daborn, G.R., 1998. El Río de la Plata. Una revisión ambiental. Un informe de antecedentes del proyecto EcoPlata. Halifax: Dalhousie University.

Zar, J.H., 1999. Biostatistical analysis. New Jersey: Prentice Hall. 